\title{
The Acceptability and Visual Impact of $0.01 \%$ Atropine in a Caucasian Population
}

James Loughman

Technological University Dublin, james.loughman@tudublin.ie

Ian Flitcroft

Mater Hospital

Follow this and additional works at: https://arrow.tudublin.ie/otpomart

Part of the Optometry Commons

\section{Recommended Citation}

Loughman J, Flitcroft DI. Br J Ophthalmol 2016;0:1-5. doi:10.1136/bjophthalmol-2015-307861

This Article is brought to you for free and open access by ARROW@TU Dublin. It has been accepted for inclusion in Articles by an authorized administrator of ARROW@TU Dublin. For more information, please contact arrow.admin@tudublin.ie, aisling.coyne@tudublin.ie, gerard.connolly@tudublin.ie.






\title{
The acceptability and visual impact of $0.01 \%$ atropine in a Caucasian population
}

\author{
James Loughman, ${ }^{1,2}$ DI Flitcroft ${ }^{3}$
}

${ }^{1}$ Department of Optometry, School of Physics, College of Sciences \& Health, Dublin Institute of Technology, Dublin, Ireland

${ }^{2}$ Faculty of Health Sciences, African Vision Research Institute, University of KwaZulu-Natal, Durban, South Africa

${ }^{3}$ Department of Ophthalmology, Mater Misericordiae University Hospital, Dublin, Ireland

\section{Correspondence to} Professor James Loughman, Department of Optometry, School of Physics, College of Sciences \& Health, Dublin Institute of Technology, Dublin, D08 NF82, Ireland; james.loughman@dit.ie

Received 17 September 2015 Revised 5 January 2016 Accepted 24 January 2016

To cite: Loughman J, Flitcroft DI. Br I Ophthalmol Published Online First: [please include Day Month Year] doi:10.1136/

bjophthalmol-2015-307861

\begin{abstract}
Background Myopia is a condition of enormous public health concern, affecting up to 2.5 billion people worldwide. The most effective treatment to prevent myopia progression is atropine but at the cost of accommodative paresis and mydriasis, necessitating the use of bifocal glasses. Low-dose atropine $(0.01 \%)$ has been found to be almost as effective with significantly reduced side effects. Since there are well-recognised differences in the effect of atropine between heavily pigmented Asian eyes and Caucasian eyes, this study aimed to determine the acceptability and tolerability of $0.01 \%$ atropine (by measuring visual performance and quality of life) as a treatment for myopia control in a Caucasian population exhibiting light irides.

Methods 14 university students aged 18-27 were recruited to the study. Participants received one drop of $0.01 \%$ atropine daily into each eye over 5 days. A range of physiological, functional and quality of life measures were assessed at baseline, day 3 and day 5 .

Results The effect of atropine was statistically significant for pupil size $(p=0.04)$ and responsiveness $(p<0.01)$. While amplitude of accommodation reduced, the change was not statistically significant. Visual acuity (distance and near) and reading speed were not adversely affected. While there was a slight increase in symptoms such as glare, overall there was no quality of life impact associated with the use of low-dose atropine. Conclusions Overall, $0.01 \%$ of atropine was generally well tolerated bilaterally and no serious adverse effects were observed. Therefore this dose appears to provide a viable therapeutic option for myopia control among Caucasian eyes.
\end{abstract}

\section{INTRODUCTION}

Myopia has now reached pandemic proportions and is expected to affect a staggering 2.5 billion people by $2020 .{ }^{1}$ Yet standard clinical care today treats only the optical and medical consequences of this condition, rather than addressing its underlying biological basis. In developed countries in East and Southeast Asia, the prevalence of myopia has doubled in the past 30 years to over $90 \%$ of young adults in some countries. ${ }^{2}$ Similar extraordinary increases in prevalence are affecting Western society, with myopia prevalence doubling to almost $50 \%$ among school-leavers in the USA ${ }^{3}$ and over $50 \%$ in parts of Europe, ${ }^{4}$ including the UK. ${ }^{5}$ The existing and future public health implications of myopic progression and of increasing myopia prevalence are considerable.

The primary risks associated with myopia progression relate to its associations with ophthalmic disease and blindness. Epidemiological studies indicate that myopia is second only to age as a risk factor for many of the major eye diseases, including glaucoma, cataract and retinal detachment, ${ }^{6}$ and is the primary risk factor in myopic maculopathy. ${ }^{7}$ Even outside Asia, myopic maculopathy is one of the leading causes of blindness among working-age people, including in the UK, ${ }^{8}$ Ireland, ${ }^{9}$ Israel ${ }^{10}$ and across other parts of Europe. ${ }^{11-13}$ Atrophic myopic maculopathy is unique, however, in having no forms of available treatment, no current treatment trials and a proven aetiological basis.

The incidence of retinal detachment has been observed to increase over 45\% in Scotland over a 20 -year period, a phenomenon that, although unproven, may well be linked to the evolving profile of myopia. ${ }^{14}$ In terms of overall risk, mild levels of myopia appear to be as dangerous to ocular health as smoking and hypertension are to cardiovascular health. ${ }^{6}$ Although myopia is not currently considered by clinicians to be a modifiable risk factor for blindness, the evidence is compelling that the introduction of a safe and effective method of reducing myopic progression would have a significant public health benefit.

A recent Cochrane review of the interventions for myopia control reported that there is no conclusive evidence that optical control modalities such as undercorrection, multifocal spectacles or contact lenses yield benefits in terms of reductions in the rate of progression, and may, in some instances, exacerbate the rate of myopia progression. ${ }^{15}$ Recent evidence suggests, however, that contact lenses which correct for peripheral hyperopia may have a clinically significant benefit for myopia control. ${ }^{16}{ }^{17}$ The Cochrane review concluded that topical antimuscarinic medication is the most likely effective treatment to slow myopia progression. ${ }^{15}$

Atropine was originally used for myopia control at its standard $1 \%$ dose, and while this proved highly effective in reducing the rate of axial elongation and myopia progression, it was, unsurprisingly, associated with the expected side effects, including pupillary dilatation, photophobia, cycloplegia and allergy. ${ }^{18}$ The most recent evidence (ATOM2 study), however, in relation to the relative efficacy of various concentrations of atropine, suggests that, over a 5 -year follow-up period, a low-dose $0.01 \%$ concentration actually exhibits the highest efficacy in slowing myopia progression with less visual side effects compared with higher doses of atropine. ${ }^{19}$ Such a low dose may, therefore, prove more tolerable and acceptable to patients as a means of effective myopia control. The ATOM2 study of low-dose atropine was conducted in Singapore on a population with heavily pigmented irides. Since race and iris colour are known factors 
that influence cycloplegia, one valid concern about applying such treatment in lightly pigmented Caucasian eyes is that the side effect profile may be more severe. ${ }^{20}$

This study has been designed, therefore, to assess the feasibility of low-dose atropine $(0.01 \%)$ for use as a potential myopia control agent in a Caucasian population exhibiting lightly pigmented irides. Specifically, the effect of low-dose atropine on (1) functional vision measures including visual acuity (VA) and reading speed; (2) physiological measures including amplitude of accommodation (AA), pupil size and convergence; and (3) vision-related quality of life were the primary objectives of the study.

\section{MATERIALS AND METHODS}

University students were invited to participate in this study, which was approved by the Research Ethics Committee at Dublin Institute of Technology. Written informed consent was obtained from all subjects, and the study adhered to the tenets of the Declaration of Helsinki. Inclusion criteria were age $18+$ years, VA better than $\log$ MAR 0.3 , light irides, refractive error ranging from emmetropia to $6 \mathrm{D}$ of myopia, Caucasian race, AA $>6 \mathrm{D}$ and normal systemic and ocular health. Volunteers were to be excluded on the basis of any evidence of a narrow anterior chamber drainage angle, known allergy to atropine or any systemic condition contraindicating the use of atropine.

Demographic information, including age and gender, were recorded for each participant. Iris colour was assessed under ambient room illumination, and eligibility confirmed under slit lamp biomicroscope illumination (absence of any conspicuous brown iris melanin pigmentation).

Non-cycloplegic autorefraction (and focimetry of existing spectacle prescription where relevant) was performed to provide an estimate of refractive error (Topcon TRK-2P Auto Kerato-Refractor). Distance VA was measured using the early treatment of diabetic retinopathy study (ETDRS) logMAR chart at $6 \mathrm{~m}$. Test letters were randomised on each visit. Near VA was measured with a reduced logMAR reading chart held at $40 \mathrm{~cm}$. AA was measured monocularly and binocularly and the near point of convergence (NPC) was measured binocularly with a Royal Air Force rule as the average of three measurements. Reading speed was assessed using the Wilkins Rate of Reading Test, which was recorded as the number of correctly identified words per second (WPS). Four separate passages of text were used to avoid any bias due to familiarity with the text. All of the above measurements were taken with distance spectacle correction worn where appropriate.

Pupil size was measured using the Neuroptics VIP-200 Pupillometer. The direct pupillary response was checked using an light emitting diode (LED) pen torch, and recorded on a scale ranging from 0 (non-responsive) to 3 (brisk).

Atropine $0.01 \%$ was prepared by dilution of unpreserved atropine 1\% minims (Bausch \& Lomb) into a well-tolerated ocular vehicle (Tears Naturale, Alcon Laboratories).

On completion of all baseline tests, one drop of $0.01 \%$ atropine was instilled in both eyes of eligible participants. Participants returned to the clinic at the same time each day for repeat instillation of the eyedrops on four subsequent days. Each of the baseline tests were repeated on two subsequent days, yielding a total of three sets of measurements for each participant (baseline (day 1), day 3, final (day 5)).

Participants also completed the visual function-14 quality of life questionnaire at baseline and on the final visit to explore the presence of any difficulties affecting routine vision-related activities. Subjects were required to assign their level of difficulty with each of the 14 vision-related tasks into one of the following grades: none, a little, moderate, great deal and unable to do. At the final visit, participants were also asked the generic question, 'Was the use of atropine eyedrops (a) acceptable/tolerable to you as a therapy for myopia control (yes or no?) and (b) compatible with the continuation of normal vision-related activities such as reading and studying (yes or no?)'.

\section{Statistical analysis}

All statistical analysis was performed using the statistical software package SPSS (V.22.0; IBM, New York, New York, USA). The Shapiro-Wilk's test was used to determine normality of the data distributions. A one-way repeated measures analysis of variance (RM-ANOVA), including Greenhouse-Geisser correction for violations of sphericity and post-hoc tests where appropriate, was used to explore the effect of the atropine preparation on measures of visual function across the duration of the study. A statistical significance level of 5\% was adopted throughout the analysis.

\section{RESULTS}

A total of 14 participants were enrolled in the study, with equal gender representation and comprising 11 myopes (refractive error range -0.75 to $-3.25 \mathrm{D}$ spherical equivalent) and three emmetropes. The mean (SD) age of participants was 22 (3).

\section{Ocular physiology}

On average, photopic pupil size increased by $1.31 \mathrm{~mm}$ (OD, right eye) and $1.08 \mathrm{~mm}$ (OS, left eye). The degree of change in pupil size was largest initially, with a $13.1 \%-18.9 \%$ increase between the baseline and day 3 measures, which then moderated to a $4.1 \%-4.9 \%$ further increase between day 3 and day 5. RM-ANOVA revealed that the change in pupil size was statistically significant in each eye $(\mathrm{p}=0.04)$. Post-hoc analysis using a paired $t$ test revealed that the pupil size at the final visit was statistically significantly different from baseline $(p=0.04)$, but not from the day 3 measure $(p=0.79)$. Pupillary response also became statistically significantly more sluggish by the final visit $(\mathrm{p}<0.01)$. The NPC receded slightly at the final visit relative to baseline, but the difference was not statistically significant $(p=0.25)$. Binocular AA exhibited an $11 \%$ reduction over the study duration (figure 1 ), but the change was not statistically significant $(\mathrm{p}=0.08)$.

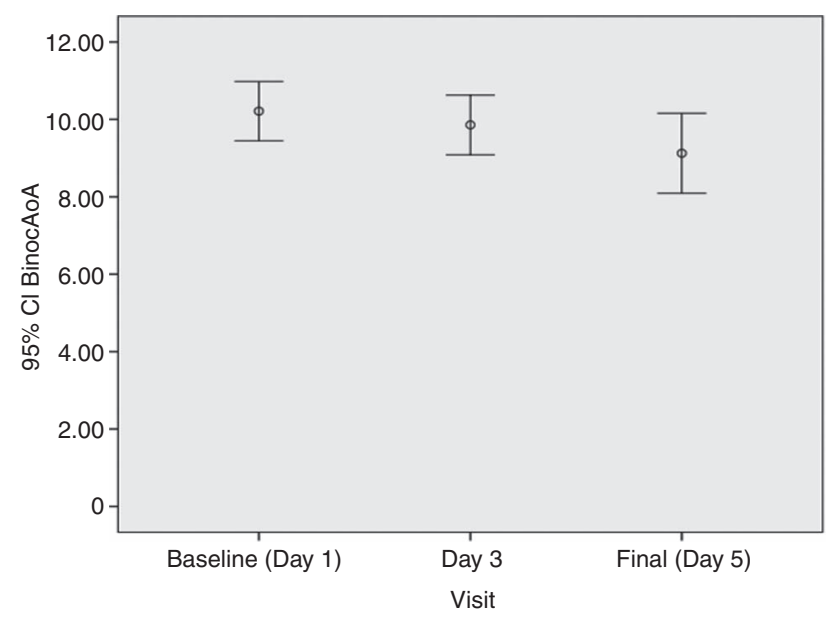

Figure 1 Change in amplitude of accommodation during the course of the study. 


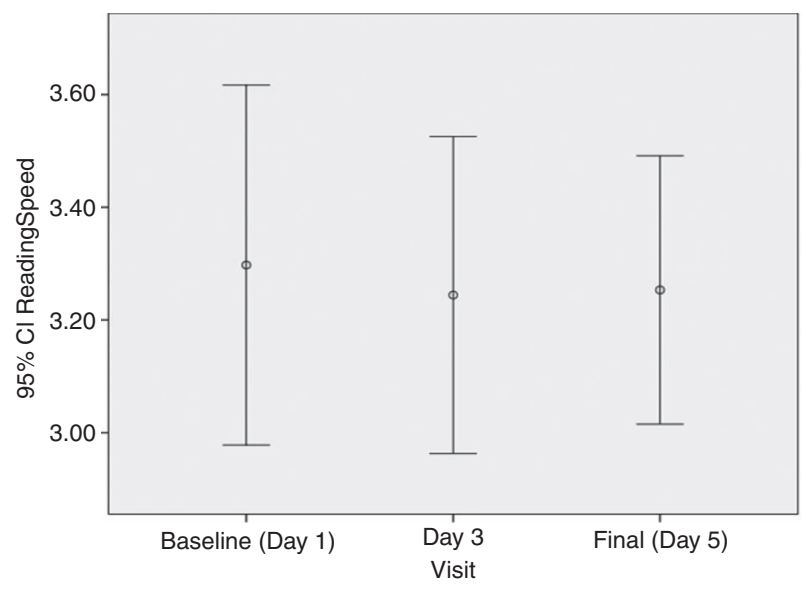

Figure 2 Change in reading speed during the course of the study.

\section{Visual function}

Reading speed remained essentially unchanged throughout the study (figure 2), ranging from 3.29 WPS at baseline to 3.25 WPS at completion $(p=0.45)$. Furthermore, distance VA exhibited only a one-letter change that was not statistically significant, while near VA remained unchanged throughout.

The mean (SD) of all clinical measurements at baseline, and across all three subsequent study visits, along with the RM-ANOVA are presented in table 1.

\section{Quality of life}

At baseline, all 14 participants reported no difficulty with any of the 14 vision-related activities contained in the visual function-14 questionnaire. At the final visit four subjects remained entirely asymptomatic. Eight subjects reported a onegrade increase in their level of difficulty (from 'none' to 'a little') with one of the 14 vision-related tasks, while two subjects reported an identical one-grade increase in their level of difficulty in two of the 14 vision-related tasks. Glare was the most commonly reported symptom by six subjects.

In response to the additional quality of life questions, all 14 reported that (1) atropine was acceptable/tolerable to them as a form of therapy for myopia control and (2) atropine was compatible with the continuation of normal vision-related activities.

\section{DISCUSSION}

As well as being effective, any intervention that requires treatment over an extended period of time must also be well tolerated. The results of this study show that low-dose atropine has minimal impact on accommodation, reading speed and visually dependent daily activities. The age profile of this study was higher than in the ATOM2 study (age group: 6-12 years), but the age-related reduction in AA means that older subjects are more sensitive to the cycloplegic side effects of low-dose atropine. The inclusion of university students, at a time of year when course exams were ongoing, meant that the visual demands of study participants were particularly exacting, more so than would be typical of younger children. In addition, the higher rate of adult-onset myopia in Europe relative to Asia requires that treatments (if available) be applied to older subjects than were recruited to the ATOM2 study.

Atropine is a competitive and non-selective antagonist of the muscarinic acetylcholine receptors. In ophthalmic use, topical atropine is used as a cycloplegic, to temporarily paralyse the accommodation reflex, and as a mydriatic, to dilate the pupils. Atropine at standard doses reaches peak cycloplegia within $1 \mathrm{~h}$ and degrades slowly, wearing off in 7-14 days, and so it is generally used as a therapeutic agent rather than as a diagnostic drug, for example, in the treatment of anterior uveitis or amblyopia in children. Despite established merits for myopia control, the adverse effects associated with the standard 1\% dose of atropine (eg, blurred near vision which is exploited as a monocular penalisation technique in amblyopia treatment) have inhibited its emergence as a viable control therapy. ${ }^{15}$

A serendipitous discovery in a trial conducted in Singapore has provided a solution to the side effects observed at the $1 \%$ dose, by demonstrating that low-dose atropine $(0.01 \%)$ remains effective at reducing myopic progression, while having minimal impact on pupil size and range of accommodation. ${ }^{19} 2122$ The efficacy of the $0.5 \%$ and $0.1 \%$ dose was effectively equal to the $1 \%$ standard dose. Myopia progression after 2 years in the $1.0 \%, 0.5 \%$ and $0.1 \%$ groups was minimal at $-0.29 \mathrm{D}$, $-0.30 \mathrm{D}$ and $-0.38 \mathrm{D}$, respectively, while the progression in the $0.01 \%$ group was not statistically significantly different at $0.49 \mathrm{D}$. That $0.01 \%$ remains effective relates to the very high level of affinity atropine has to muscarinic receptors. At normal clinical doses atropine blocks all receptor subtypes (M1-M5), but the $0.01 \%$ dose has minimal impact on pupil size and

Table 1 Impact of low-dose $(0.01 \%)$ atropine on measures of ocular physiology and visual function

\begin{tabular}{|c|c|c|c|c|}
\hline Variable & Baseline (day 1) & Day 3 & Final (day 5) & $\begin{array}{l}\text { RM-ANOVA } \\
\text { p Value }\end{array}$ \\
\hline Distance VA OD & $-0.10(0.10)$ & $-0.08(0.07)$ & $-0.08(0.05)$ & 0.547 \\
\hline Distance VA OS & $-0.10(0.07)$ & $-0.08(0.06)$ & $-0.08(0.05)$ & 0.644 \\
\hline Near VA OD & N5 & N5 & N5 & - \\
\hline Near VA OS & N5 & N5 & N5 & - \\
\hline AA OD & $9.5 \mathrm{D}(1.78)$ & $9.3 \mathrm{D}(1.44)$ & $9.1 \mathrm{D}(2.83)$ & 0.18 \\
\hline AA OS & $9.3 \mathrm{D}(2.14)$ & $9.8 \mathrm{D}(1.94)$ & 8.6 D (1.90) & 0.11 \\
\hline AA OU & $10.2 \mathrm{D}(1.42)$ & $10 \mathrm{D}(1.39)$ & $9.1 \mathrm{D}(2.12)$ & 0.08 \\
\hline Pupil size $(\mathrm{mm})$ OD & $5.51(1.74)$ & $6.55(1.12)$ & $6.82(0.61)$ & 0.04 \\
\hline Pupil size $(\mathrm{mm})$ OS & $5.81(1.73)$ & $6.57(1.32)$ & $6.89(0.92)$ & 0.04 \\
\hline Reading speed (WPS) & $3.36(0.55)$ & $3.16(0.54)$ & $3.25(0.45)$ & 0.12 \\
\hline Pupillary responses & $3.0(0.00)$ & $2.8(0.45)$ & $1.1(0.90)$ & 0.00 \\
\hline $\mathrm{NPC}(\mathrm{cm})$ & $6.8(2.43)$ & $7.0(2.19)$ & 8. (3.24) & 0.25 \\
\hline
\end{tabular}

Statistically significant findings are highlighted in bold text.

AA, amplitude of accommodation; NPC, near point of convergence; OD, right eye; OS, left eye; OU, both eyes; RM-ANOVA, repeated measures analysis of variance; VA, visual acuity; WPS, words per second. 
accommodation (M3 receptors), ${ }^{23}$ while retaining most of its beneficial effects on myopic progression (M1 and M4). ${ }^{24}$ An additional advantage of the low-dose atropine is that a rebound acceleration of myopia progression observed on cessation of $1 \%$ atropine treatment is not observed at this $0.01 \%$ concentration. $^{22}$ The benefit is such that after 5 years of follow-up, the progression of myopia is actually least in the $0.01 \%$ group relative to all higher concentrations. ${ }^{19}$ A recent retrospective casecontrol study has also reported that atropine $0.01 \%$ eyedrops significantly reduced the rate of myopic progression over 1 year with minimal side effects (progression $-0.1 \mathrm{D}$ compared with $-0.6 \mathrm{D}$ for controls). This study suggests that the low dose appears most effective in children with low initial myopia but may not control rapid myopic progression in some patients. ${ }^{25}$

Topical antimuscarinic drugs are unusual in that there is a well-described racial variation in sensitivity to such drugs that relates to the amount of pigmentation (specifically melanin) within the iris. ${ }^{20} 26$ Iris melanin content is important in the context of atropine use as melanin is known to sequester anticholinergic compounds. ${ }^{27}$ An eye with dark brown irides has two to four times more ocular melanin than one with blue irides. ${ }^{28}$ The effect is such that the same concentration of drug has less cycloplegic and mydriatic effect on darker relative to lighter irides. Melanin-bound atropine is slowly released such that recovery from the effect on accommodation and pupil size is longer seen in darker irides. The ability of melanin to bind and alter the pharmacokinetics of a drug such as atropine may have an impact on the tolerability or efficacy of the therapy, and therefore limits the extrapolation of results from a trial conducted in Asia to Europe. While one might expect increased efficacy of atropine in Caucasian eyes, there is also the concern that the possible side effects might be worse. Such racial differences may, therefore, reduce the acceptability of the therapy in populations with a higher proportion of Caucasians.

The physiological, functional and quality of life impact of low-dose atropine in a Caucasian population are critical in terms of the future acceptability of atropine as a viable therapeutic intervention. Importantly, this study included only those with light irides of most concern from a safety and tolerability perspective.

As expected, the use of atropine was associated with physiological changes such as pupillary dilatation, decreased pupillary response and accommodative amplitude. The magnitude of such changes appears small and was not associated with any detectable loss of functional vision. Reading speed and VA, both distance and near, remained essentially unchanged throughout the study.

There was, however, some quality of life impact associated with the physiological response to the low-dose atropine. Only four participants remained entirely asymptomatic, and it is unsurprising to note that glare, presumably associated with the reduced pupillary responsiveness and increase in pupil size, was the most frequently cited quality of life impact. The impact again was minimal, and perhaps the most important finding of this pilot investigation is that all 14 participants found the use of low-dose atropine tolerable, and it did not adversely impact the continuation of their exacting visual activities. These results are compatible with those obtained among Asian participants with darker irides. In particular, the $0.01 \%$ dose employed in the ATOM2 trial was not associated with the side effects typical of the standard clinical dose. ${ }^{19} 21$ Even the reduced $0.5 \%$ and $0.1 \%$ doses employed in ATOM2 were associated with a negative impact on participant quality of life, with $70 \%$ and $61 \%$ of participants on these respective doses requesting the prescription of progressive addition lenses to assist their reading during the study, compared with only $6 \%$ in the $0.01 \%$ group. More recently, a $0.02 \%$ concentration has been established as the maximum atropine dose that can be administered in Asian eyes without a clinical impact on vision. ${ }^{29}$

The results of this study indicate, therefore, that $0.01 \%$ of atropine instilled bilaterally over a period of 5 days was generally well tolerated without serious adverse effects. While these findings are limited in terms of sample size and duration, we would suggest that, as a pilot investigation, they are sufficient to confirm the acceptability and minimal side effect profile of low-dose atropine for myopia control in a Caucasian population likely to exhibit lighter irides than is typical in Asia.

Acknowledgements The authors wish to thank Valerie Duignan, Ronan Keogh, Chloe Fennell and Jessica Dolan for their work in the data collection phase of the project.

Contributors $\mathrm{J}$ contributed to the conception and design of the study, data capture, analysis and interpretation. He drafted the initial paper, approved the final version and agrees to be accountable for all aspects of the work in ensuring that questions related to the accuracy or integrity of any part of the work are appropriately investigated and resolved. DIF contributed to the conception and design of the study, and interpretation of study findings. He reviewed the initial paper and provided recommendations for change. He also approved the final version and agrees to be accountable for all aspects of the work in ensuring that questions related to the accuracy or integrity of any part of the work are appropriately investigated and resolved.

Competing interests None declared.

Patient consent Obtained.

Ethics approval Research Ethics Committee, Dublin Institute of Technology.

Provenance and peer review Not commissioned; externally peer reviewed.

Data sharing statement The data is stored electronically on an encrypted system, and hard copy in a locked filing cabinet. Only the two investigators have access to the study data.

\section{REFERENCES}

1 Morgan IG, Ohno-Matsui K, Saw SM. Myopia. Lancet 2012;379:1739-48.

2 Pan CW, Ramamurthy D, Saw SM. Worldwide prevalence and risk factors for myopia. Ophthalmic Physiol Opt 2012;32:3-16.

3 Vitale S, Sperduto RD, Ferris FL III, et al. Increased prevalence of myopia in the United States between 1971-1972 and 1999-2004. Arch Ophthalmol 2009;127:1632-9.

4 Wolfram C, Hohn R, Kottler U, et al. Prevalence of refractive errors in the European adult population: the Gutenberg Health Study (GHS). Br J Ophthalmol 2014; 55:2041-7

5 Logan NS, Davies LN, Mallen EA, et al. Ametropia and ocular biometry in a U.K. university student population. Optom Vis Sci 2005;82:261-6.

6 Flitcroft DI. The complex interactions of retinal, optical and environmental factors in myopia aetiology. Prog Ret Eye Res 2012;31:622-60.

7 Hayashi K, Ohno-Matsui K, Shimada N, et al. Long-term pattern of progression of myopic maculopathy: a natural history study. Ophthalmology 2010;117:1595-611, 1611.e1-4.

8 Evans JR, Fletcher AE, Wormald RPL, et al. Causes of visual impairment in people aged 75 years and older in Britain: an add-on study to the MRC Trial of Assessment and Management of Older People in the Community. Br J Ophthalmol 2004;88:365-70

9 Kelliher C, Kenny D, O'Brien C. Trends in blind registration in the adult population of the Republic of Ireland 1996-2003. Br J Ophthalmol 2006;90:367-71.

10 Avisar R, Friling R, Snir M, et al. Estimation of prevalence and incidence rates and causes of blindness in Israel, 1998-2003. Isr Med Assoc J 2006;8:880-1.

11 Cedrone C, Nucci C, Scuderi G, et al. Prevalence of blindness and low vision in an Italian population: a comparison with other European studies. Eye (Lond) 2006;20:661-7

12 Buch $H$, Vinding T, La Cour M, et al. Prevalence and causes of visual impairment and blindness among 9980 Scandinavian adults: the Copenhagen City Eye Study. Ophthalmology 2004;111:53-61.

13 Klaver CC, Wolfs RC, Vingerling JR, et al. Age-specific prevalence and causes of blindness and visual impairment in an older population: the Rotterdam Study. Arch Ophthalmol 1998;116:653-8.

14 Mitry D, Chalmers J, Anderson K, et al. Temporal trends in retinal detachment incidence in Scotland between 1987 and 2006. Br J Ophthalmol 2011; 95:365-9. 
15 Walline JJ, Lindsley K, Vedula SS, et al. Interventions to slow progression of myopia in children. Cochrane Database Syst Rev 2011;(12):CD004916.

16 Lam CSY, Tang WC, Tse DY-Y, et al. Defocus Incorporated Soft Contact (DISC) lens slows myopia progression in Hong Kong Chinese schoolchildren: a 2-year randomised clinical trial. Br J Ophthalmol 2014;98:40-5.

17 Sankaridurg P, Holden B, Smith EL III, et al. Decrease in rate of myopia progression with a contact lens designed to reduce relative peripheral hyperopia: one-year results. Invest Ophthalmol Vis Sci 2011:52:9362-7.

18 Chua WH, Balakrishnan V, Chan YH, et al. Atropine for the treatment of childhood myopia. Ophthalmology 2006;113:2285-91.

19 Chia A, Lu QS, Tan D. Five-year clinical trial on atropine for the treatment of myopia 2: Myopia control with atropine $0.01 \%$ eyedrops. Ophthalmology 2016;123:391-9.

20 Manny RE, Hussein M, Scheiman M, et al, The COMET Study Group. Tropicamide (1\%): An effective cycloplegic agent for myopic children. Invest Ophthalmol Vis Sci 2001;42:1728-35.

21 Chia A, Chua WH, Cheung YB, et al. Atropine for the treatment of childhood myopia: safety and efficacy of $0.5 \%, 0.1 \%$, and $0.01 \%$ doses (Atropine for the Treatment of Myopia 2). Ophthalmology 2012;119:347-54.
22 Chia A, Chua WH, Wen L, et al. Changes after cessation of atropine: ATOM2. Am J Ophthalmol 2014;157:451-457.e1.

23 Gil DW, Krauss HA, Bogardus AM, et al. Muscarinic receptor subtypes in human iris-ciliary body measured by immunoprecipitation. Invest Ophthalmol Vis Sci 1997;38:1434-42.

24 Arumugam B, McBrien NA. Muscarinic antagonist control of myopia: evidence for M4 and M1 receptor-based pathways in the inhibition of experimentally-induced axial myopia in the tree shrew. Invest Ophthalmol Vis Sci 2012;53:5827-37.

25 Clark TY, Clark RA. Atropine $0.01 \%$ eyedrops significantly reduce the progression of childhood myopia. J Ocul Pharmacol Ther 2015;31:541-5.

26 Koneru PB, Lien EJ, Koda RT. Review: Oculotoxicities of systemically administered drugs. J Ocular Pharm 1986;2:385-99.

27 Salazar-Bookaman MM, Wainer I, Patil PN. Relevance of drug-melanin interactions to ocular pharmacology and toxicology. I Ocul Pharmacol 1994;10:217-39.

28 Ray K, Chaki M, Sengupta M. Tyrosinase and ocular diseases: Some novel thoughts on the molecular basis of oculocutaneous albinism type 1. Prog Retin Eye Res 2007;26:323-58

29 Cooper J, Eisenberg N, Schulman E, et al. Maximum atropine dose without clinical signs or symptoms. Optom Vis Sci 2013;90:1467-72. 


\section{The acceptability and visual impact of $0.01 \%$ atropine in a Caucasian population}

James Loughman and DI Flitcroft

Br J Ophthalmol published online February 22, 2016

Updated information and services can be found at:

http://bjo.bmj.com/content/early/2016/02/22/bjophthalmol-2015-30786

1

These include:

References This article cites 28 articles, 8 of which you can access for free at: http://bjo.bmj.com/content/early/2016/02/22/bjophthalmol-2015-30786 1\#BIBL

Email alerting Receive free email alerts when new articles cite this article. Sign up in the service box at the top right corner of the online article.

Topic Articles on similar topics can be found in the following collections

Collections

Optic nerve (699)

Optics and refraction (500)

Neurology (1314)

Pupil (51)

Public health (469)

\section{Notes}

To request permissions go to:

http://group.bmj.com/group/rights-licensing/permissions

To order reprints go to:

http://journals.bmj.com/cgi/reprintform

To subscribe to BMJ go to:

http://group.bmj.com/subscribe/ 